\title{
TOPOLOGY AND FLUX OF T-DUAL MANIFOLDS WITH CIRCLE ACTIONS
}

\author{
VARGHESE MATHAI AND SIYE WU
}

\begin{abstract}
We present an explicit formula for the topology and H-flux of the T-dual of a general type II compactification, significantly generalizing earlier results. Our results apply to T-dualities with respect to any circle action on spacetime $X$. As before, T-duality exchanges type IIA and type IIB string theories. A new consequence is that the T-dual spacetime is a singular space when the fixed point set $X^{\mathbb{T}}$ is non-empty; the singularities correspond to Kaluza-Klein monopoles. We propose that the Ramond-Ramond charges of type II string theories on the singular dual are classified by twisted equivariant cohomology groups. We also discuss the K-theory approach.
\end{abstract}

It is by now well known that there are five (super) string theories related to each other by transformations called dualities. Two string theories are said to be dual to each other if they are equivalent descriptions of the same theory at the quantum level.

The global aspects of one such duality, called T-duality in type II string theories, involves compactifications of spacetime $X$ with an H-flux. It is a generalization of the $R \rightarrow 1 / R$ transformation of string theories compactified on a circle of radius $R$. The local transformation rules of the low energy effective fields under T-duality, known as the Buscher rules [1], have been known for some time. However, in cases in which there is a topologically nontrivial NS 3-form H-flux, the Buscher rules only make sense on each local spacetime patch. Several examples of T-duals to such backgrounds have been found [2, 3, 4, 5, 6] and in each case it was seen that T-duality changes not only the H-flux but also the spacetime topology. In [7], a general formula was presented for the topology and H-flux of a compactification from the topology and H-flux of its T-dual, with respect to any free circle action, in particular reproducing the examples in the literature mentioned above. As supporting evidence, it was shown there that locally, the said formula agrees with the Buscher rules and that globally it yields an isomorphism of the twisted cohomology and twisted K-theory-valued conserved Ramond-Ramond charges [8, 9, 10, 11, 12.

In this paper, we will produce an explicit formula for the topology and H-flux of the T-dual of a more general type II compactification, significantly generalizing earlier results. Our results apply

\footnotetext{
Acknowledgements. This research was supported under Australian Research Council's Discovery Projects funding scheme (project number DP0878184). V.M. is the recipient of an Australian Research Council Australian Professorial Fellowship (project number DP0770927). S.W. is supported in part by a General Research Fund from the Research Grants Council of Hong Kong (project number HKU706010P). The authors would like to thank Jarah Evslin and Jonathan Rosenberg for useful feedback.
} 
to T-dualities with respect to any circle action. We start with the spacetime $X$, equipped with a flux form $H$, that is a manifold without boundary such that the fixed point set $X^{\mathbb{T}}$ is non-empty. The T-dual spacetime $\widehat{X} / \mathbb{T}$ is a stratified space, possibly with boundary, in the sense of [13], with a dual flux $\widehat{H}$ which is an equivariantly closed differential form on $\widehat{X}$. The singularities of the spacetime and that of the flux form correspond to Kaluza-Klein monopoles [14, 12]. Topological aspects of examples from mirror symmetry (when $X$ is Calabi-Yau, cf. [15]) fit into our framework, solving an open problem stated in [7]. Technically, the new approach in this paper is to use the correspondence space in a fundamental way in our construction of the T-dual spacetime, in contrast with the construction of the T-dual in [7]. We show that the twisted cohomology of $(X, H)$ is isomorphic, with a degree shift, to the twisted equivariant cohomology of $(\widehat{X}, \widehat{X})$, and a similar result in twisted equivariant $\mathrm{K}$-theory. We thus propose that the Ramond-Ramond charges of type II strings theories on the singular space $\widehat{X} / \mathbb{T}$, with the flux form $\widehat{H}$, are classified by the twisted equivariant cohomology groups. Possible relevance of equivariant cohomology in the context of Tduality was first mentioned in $\S 2$, [16]. Intersection cohomology [13] is another good replacement for the cohomology of $\widehat{X} / \mathbb{T}$, and it is interesting to see if it is possible to realize T-duality isomorphisms in terms of intersection cohomology and its twisted variants.

Consider a pair $(X, H)$, where $X$ is a spacetime and $H$ is a background flux, a closed differential 3 -form on $X$ with integral periods. We also suppose that there is a smooth action of a circle $\mathbb{T}$ on $X$ preserving $H$ (by averaging over $\mathbb{T}$, this can always be arranged without changing the cohomology class of $H)$. We now construct the T-dual of $(X, H)$ in such a way that it reduces to the case studied in [7] whenever the $\mathbb{T}$-action is free. Consider the product $X \times E \mathbb{T}$ with the diagonal $\mathbb{T}$-action, which is free since the action on the universal space $E \mathbb{T}$ is free. The universal $\mathbb{T}$-space $E \mathbb{T}$ can be chosen to be either the unit sphere in an infinite dimensional Hilbert space, or the direct limit of odd dimensional spheres $S^{\infty}=\lim _{\longrightarrow} S^{2 n+1}$. Therefore $X \times E \mathbb{T}$ is a smooth countably compactly generated manifold and $p_{1}^{*}(H)$, where $p_{1}: X \times E \mathbb{T} \rightarrow X$ is the projection, is a background flux on it. The quotient space

$$
X_{\mathbb{T}}=(X \times E \mathbb{T}) / \mathbb{T}
$$

where $\mathbb{T}$ acts diagonally on $X \times E \mathbb{T}$, is called the Borel construction. Note that there is a fibration

$$
X \hookrightarrow X_{\mathbb{T}} \longrightarrow B \mathbb{T}
$$

where the classifying space $B \mathbb{T}$ can be chosen to be either the projective Hilbert space, or the direct limit of complex projective spaces $\mathbb{C} P^{\infty}=\lim _{\longrightarrow} \mathbb{C} P^{n}$. The equivariant cohomology is by fiat $\mathrm{H}_{\mathbb{T}}^{\bullet}(X, \mathbb{Z})=\mathrm{H}^{\bullet}\left(X_{\mathbb{T}}, \mathbb{Z}\right)$. It has the following properties, cf. [17].

(1) The equivariant cohomology $\mathrm{H}_{\mathbb{T}}^{\bullet}(X, \mathbb{Z})$ is a module over $\mathrm{H}^{\bullet}(B \mathbb{T}, \mathbb{Z})=\mathbb{Z}[\phi]$.

(2) The inclusion map $i: X \hookrightarrow X_{\mathbb{T}}$ induces the restriction $i^{*}: \mathrm{H}_{\mathbb{T}}^{\bullet}(X, \mathbb{Z}) \rightarrow \mathrm{H}^{\bullet}(X, \mathbb{Z})$. 
(3) The surjective map $\sigma: X_{\mathbb{T}} \rightarrow X / \mathbb{T}$ induces a map $\sigma^{*}: \mathrm{H}^{\bullet}(X / \mathbb{T}, \mathbb{Z}) \rightarrow \mathrm{H}_{\mathbb{T}}^{\bullet}(X, \mathbb{Z})$, which is an isomorphism whenever the $\mathbb{T}$-action on $X$ is free.

(4) Upon localisation, there is an isomorphism $\mathrm{H}_{\mathbb{T}}^{\bullet}(X)\left[\phi^{-1}\right] \cong \mathrm{H}^{\bullet}\left(X^{\mathbb{T}}\right)\left[\phi, \phi^{-1}\right]$, where $X^{\mathbb{T}}$ denotes the fixed point subset of $X$.

These properties explain why the equivariant cohomology $\mathrm{H}_{\mathbb{T}}(X)$ is a good replacement of the cohomology of $X / \mathbb{T}$ when the latter is singular.

The Gysin sequence of the $\mathbb{T}$-fibration $X \times E \mathbb{T} \rightarrow X_{\mathbb{T}}$ is the long exact sequence

$$
\rightarrow \mathrm{H}^{j}(X) \stackrel{\pi_{*}}{\rightarrow} \mathrm{H}^{j-1}\left(X_{\mathbb{T}}\right) \stackrel{\cup e_{\mathbb{T}}}{\rightarrow} \mathrm{H}^{j+1}\left(X_{\mathbb{T}}\right) \stackrel{\pi^{*}}{\rightarrow} \mathrm{H}^{j+1}(X) \rightarrow
$$

where $\cup e_{\mathbb{T}}$ denotes the cup product with the (equivariant) Euler class $e_{\mathbb{T}} \in \mathrm{H}^{2}\left(X_{\mathbb{T}}, \mathbb{Z}\right)=\mathrm{H}_{\mathbb{T}}^{2}(X, \mathbb{Z})$. In this case, $e_{\mathbb{T}}=\phi$, which replaces the curvature of the (singular) $\mathbb{T}$-fibration $X \rightarrow X / \mathbb{T}$. So the Gysin sequence becomes

$$
\rightarrow \mathrm{H}^{j}(X) \stackrel{\pi_{*}}{\rightarrow} \mathrm{H}_{\mathbb{T}}^{j-1}(X) \stackrel{\cup \phi}{\rightarrow} \mathrm{H}_{\mathbb{T}}^{j+1}(X) \stackrel{\pi^{*}}{\rightarrow} \mathrm{H}^{j+1}(X) \rightarrow
$$

We next construct the T-dual of $(X, H)$. We recall the classification of equivariant circle bundles on a $\mathbb{T}$-manifold $X$.

Proposition 1 (Equivariant circle bundles) Let $X$ be a connected $\mathbb{T}$-manifold. The equivariant first Chern class $c_{1}^{\mathbb{T}}$ gives rise to a one-to-one correspondence between equivalence classes of $\mathbb{T}$ equivariant circle bundles over $X$ and elements of $\mathrm{H}_{\mathbb{T}}^{2}(X, \mathbb{Z})$.

For locally finite $\mathrm{CW}$-complexes with continuous $\mathbb{T}$-actions, this result was proved in [18]. For smooth actions of connected groups, Proposition 1 is due to [19]. There are different proofs, due to [20], Theorem C.47, and [21]. None of these proofs are particularly simple.

By Proposition 1, there is a $\mathbb{T}$-equivariant circle bundle $\widehat{X}$ over $X$ such that $c_{1}^{\mathbb{T}}(\widehat{X})=\pi_{*}([H]) \in$ $\mathrm{H}_{\mathbb{T}}^{2}(X, \mathbb{Z})$. The next goal is to define a class $[\widehat{H}] \in \mathrm{H}_{\mathbb{T}}^{3}(\widehat{X}, \mathbb{Z})$ corresponding to a flux on the singular space $\widehat{X} / \mathbb{T}$. Consider the commutative diagram

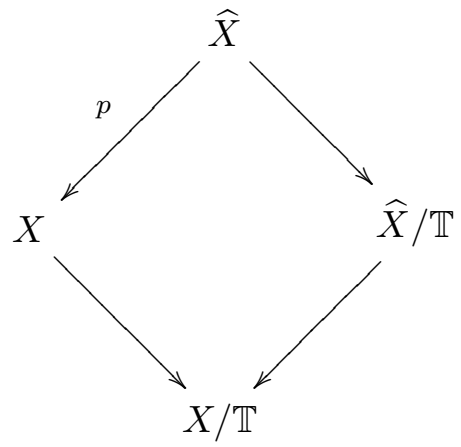


containing the singular spaces $X / \mathbb{T}$ and $\widehat{X} / \mathbb{T}$. We can replace the singular spaces by their Borel constructions by lifting the previous diagram to

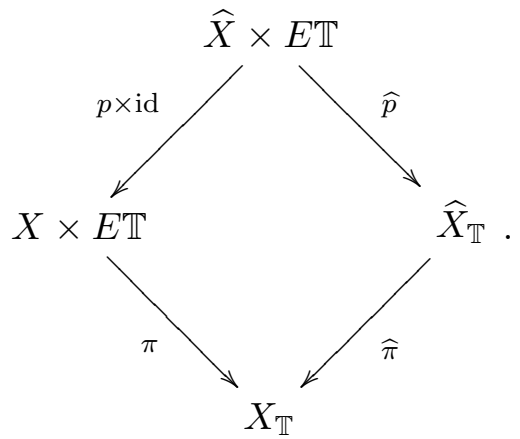

The conditions that uniquely specify $[\widehat{H}]$ are:

(1) $\widehat{\pi}_{*}([\widehat{H}])=e_{\mathbb{T}} \in \mathrm{H}_{\mathbb{T}}^{2}(X, \mathbb{Z})$;

(2) $(p \times \mathrm{id})^{*}([H])=i^{*}([\widehat{H}]) \in \mathrm{H}^{3}(\widehat{X}, \mathbb{Z})$, where $i: \widehat{X} \rightarrow \widehat{X}_{\mathbb{T}}$ is the inclusion map. That is, $[\widehat{H}]$ is an equivariant extension of $p^{*}([H])$ on $\widehat{X}$.

Recall that the Cartan model for equivariant cohomology (with real coefficients) is

$$
\mathrm{H}_{\mathbb{T}}^{\bullet}(X)=\mathrm{H}^{\bullet}\left(\Omega^{\bullet}(X)^{\mathbb{T}}[\phi], d-\phi \iota_{V}\right),
$$

where $\Omega^{\bullet}(X)^{\mathbb{T}}[\phi]$ denotes polynomials in $\phi$ with coefficients that are $\mathbb{T}$-invariant differential forms on $X$ and $V$ is the vector field on $X$ generating the $\mathbb{T}$-action. The class $c_{1}^{\mathbb{T}}(\widehat{X})=\pi_{*}([H])$ is represented by $\iota_{V} H$. On $\widehat{X} \rightarrow X$, there is a $\mathbb{T}$-invariant connection $\widehat{A} \in \Omega^{1}(\widehat{X})^{\mathbb{T}}$ whose (equivariant) curvature is $\widehat{F}=d \widehat{A}=\iota_{V} H$, with the property $\iota_{V} \widehat{A}=0$. We can choose an equivariantly closed 3-form $\widehat{H}=\widehat{A} \wedge \phi+H \in \Omega^{3}(\widehat{X})^{\mathbb{T}}[\phi]$ representing $[\widehat{H}]$. Just as the twisted cohomology $\mathrm{H}^{\bullet}(X, H)$ is the cohomology of the de Rham complex $\Omega^{\bullet}(X)$ with a twisted differential $d_{H}=d+H \wedge \cdot$, the twisted equivariant cohomology $\mathrm{H}_{\mathbb{T}}^{\bullet}(\widehat{X}, \widehat{H})$ is defined as the cohomology of the Cartan complex $\Omega^{\bullet}(\widehat{X})^{\mathbb{T}}[\phi]$ with a twisted equivariant differential $\widehat{d}_{\widehat{H}}=d-\phi \iota_{V}+\widehat{H} \wedge \cdot$. They are both only $\mathbb{Z}_{2}$-graded cohomology groups. Our main result is the following:

Theorem 1 (T-duality for circle actions) Let $X$ be a connected manifold and let $H$ be $a$ background flux, that is, $H$ is a closed differential 3-form on $X$ with integral periods. We also suppose that there is a smooth action of a circle $\mathbb{T}$ on $X$ preserving $H$. The T-dual of $(X, H)$ is the pair $(\widehat{X}, \widehat{H})$ constructed above, where $\widehat{\pi}: \widehat{X} \rightarrow X$ is a T-equivariant circle bundle over $X$ such that $c_{1}^{\mathbb{T}}(\widehat{X})=\pi_{*}([H]), \widehat{\pi}_{*}([\widehat{H}])=e_{\mathbb{T}}$ and $p^{*}([H])=i^{*}([\widehat{H}]) \in H^{3}(\widehat{X})$. There is a "T-duality isomorphism" between the twisted cohomology groups

$$
\mathrm{H}^{\bullet}(X, H) \cong \underset{4}{\mathrm{H}_{\mathbb{T}}^{\bullet+1}}(\widehat{X}, \widehat{H})
$$


We note that the T-dual of the spacetime $X$ with flux $H$ for general circle actions, is a singular manifold $\widehat{X} / \mathbb{T}$, whose desingularization $\widehat{X}$ is of dimension one higher than that of $X$. Although typically, the equivariant cohomology of a $\mathbb{T}$-manifold $X$ with nonempty fixed point set $X^{\mathbb{T}}$ is infinite dimensional, the isomorphism in Theorem 1 implies that the twisted equivariant cohomology groups $\mathrm{H}_{\mathbb{T}}^{\bullet}(\widehat{X}, \widehat{H})$ are always finite dimensional. The isomorphism in Theorem 1 is a compelling evidence that the Ramond-Ramond charges in the type II string theories on the singular space $\widehat{X} / \mathbb{T}$ are classified by the twisted equivariant cohomology group $\mathrm{H}_{\mathbb{T}}^{\bullet}(\widehat{X}, \widehat{H})$. Further evidence will be given by a similar isomorphism in twisted equivariant K-theory in the Appendix.

We explain the above isomorphism in two different ways. First, by the main result in [7] and using the commutative diagram above, we have

$$
\mathrm{H}^{\bullet}(X, H) \cong \mathrm{H}^{\bullet}\left(X \times E \mathbb{T}, p_{1}^{*}(H)\right) \cong \mathrm{H}^{\bullet+1}\left(\widehat{X}_{\mathbb{T}}, \widehat{H}\right) \cong \mathrm{H}_{\mathbb{T}}^{\bullet+1}(\widehat{X}, \widehat{H})
$$

The isomorphism can also be obtained by Hori type formulas [22], which we now establish, in the Cartan model of equivariant cohomology. Define the maps

$$
\begin{aligned}
& S: \Omega^{\bullet}(X)^{\mathbb{T}} \rightarrow \Omega^{\bullet+1}(\widehat{X})^{\mathbb{T}}[\phi], \\
& T: \Omega^{\bullet}(\widehat{X})^{\mathbb{T}}[\phi] \rightarrow \Omega^{\bullet+1}(X)^{\mathbb{T}}
\end{aligned}
$$

by

$$
\begin{aligned}
& S: G \longmapsto-\iota_{V} G+\widehat{A} \wedge G, \\
& T: \omega(\phi)=\omega_{1}(\phi)+\widehat{A} \wedge \omega_{2}(\phi) \longmapsto \omega_{2}(0),
\end{aligned}
$$

where $\omega_{1}(\phi), \omega_{2}(\phi) \in \Omega^{\bullet}(X)^{\mathbb{T}}[\phi]$. Then one verifies that

$$
\begin{aligned}
& S \circ d_{H}+\widehat{d}_{\widehat{H}} \circ S=0, \\
& T \circ \widehat{d}_{\widehat{H}}+d_{H} \circ T=0 .
\end{aligned}
$$

Moreover,

$$
\begin{aligned}
T \circ S & =\mathrm{id}, \\
\mathrm{id}-S \circ T & =R \circ \widehat{d}_{\widehat{H}}+\widehat{d}_{\widehat{H}} \circ R,
\end{aligned}
$$

where

$$
R: \Omega^{\bullet}(\widehat{X})^{\mathbb{T}}[\phi] \rightarrow \Omega^{\bullet+1}(\widehat{X})^{\mathbb{T}}[\phi]
$$

is defined by

$$
R: \omega(\phi) \longmapsto \phi^{-1}\left[\omega_{2}(\phi)-\omega_{2}(0)\right] .
$$

Therefore $S$ and $T$ are homotopy inverses of each other and the induced maps $S_{*}$ and $T_{*}$ on the twisted cohomology groups realize the isomorphism in Theorem 1. 
If we started with the spacetime $X$ without boundary and with $H=0$, the T-dual spacetime $\widehat{X} / \mathbb{T}$ is a manifold with non-empty boundary components. (In addition, there is a noncommutative algebra associated to the T-dual. See, for example, [12.) The dual flux is $\widehat{H}=\phi \wedge d \widehat{\theta}$, where $d \widehat{\theta}$ is the standard 1-form on $\widehat{\mathbb{T}}$. Near a component of the fixed-point set, $X$ looks like $\mathbb{C}^{n} \times M$, where $\mathbb{T}$ acts on each coordinate of $\mathbb{C}^{n}$ and $\operatorname{dim} M=10-2 n$. If the weights of the $\mathbb{T}$-action are 1 , the T-dual manifold $\widehat{X} / \mathbb{T}$ is the product of a cone on $\mathbb{C} P^{n-1}$ with $M \times \widehat{\mathbb{T}}$ near the singularity. For small $n$, a neighbourhood of the singularity is illustrated in Figure 1.

Figure 1. Singularity of the T-dual space of $\mathbb{C}^{n} \times M^{10-2 n}$. (a) $n=1$; (b) $n=2$;

(c) $n=3$.

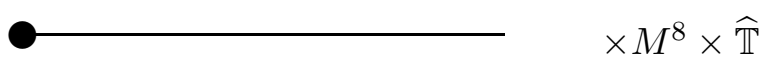

(a)
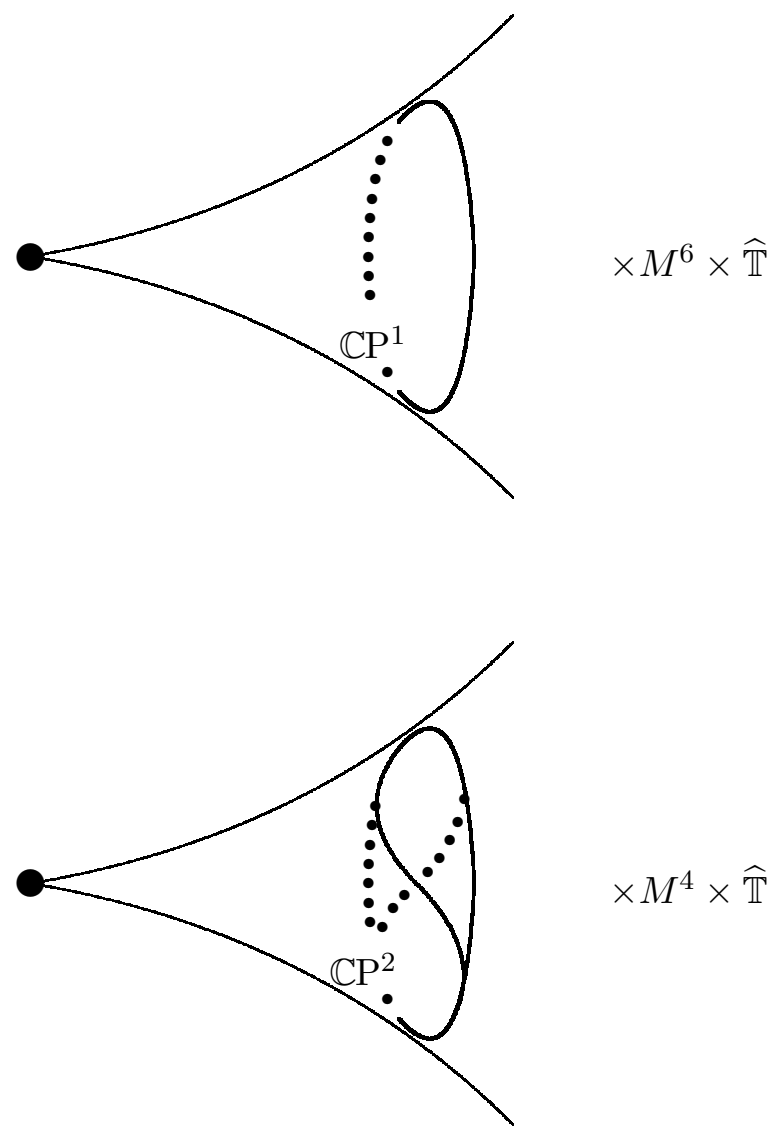

(c) 


\section{Appendix}

The appropriate K-theory for countably compactly generated spaces and $\sigma$ - $C^{*}$-algebras is representable K-theory, denoted by RK, cf. [23]. The RK-theory agrees with the usual K-theory of compact spaces and $C^{*}$-algebras for a compact space or a $C^{*}$-algebra. Many of the nice properties that K-theory satisfies generalise to RK-theory:

(1) The RK-theory is homotopy invariant and satisfies Bott periodicity, hence it is $\mathbb{Z}_{2}$-graded.

(2) If $A$ is a $C^{*}$-algebra, then there is a natural isomorphism $\operatorname{RK}_{i}(A) \cong \mathrm{K}_{i}(A)$.

(3) There is a natural isomorphism $\operatorname{RK}_{i}(A \otimes \mathcal{K}) \cong \mathrm{RK}_{i}(A)$, where $\mathcal{K}$ denotes the algebra of compact operators on a separable Hilbert space.

Let $\mathrm{CT}(X, H)$ denote the continuous trace $C^{*}$-algebra on $X$ consisting of the sections of an algebra bundle of compact operators over $X$, with Dixmier-Douady class $H$. Then the twisted K-theory is by definition [24]

$$
\mathrm{K}^{\bullet}(X, H)=\mathrm{K}_{\bullet}(\mathrm{CT}(X, H))
$$

Now $\mathrm{CT}\left(X \times E \mathbb{T}, p_{1}^{*}(H)\right)=\lim _{n} \mathrm{CT}\left(X \times S^{2 n+1}, p_{1}^{*}(H)\right)$ is a $\sigma-C^{*}$-algebra and the twisted RK-theory is

$$
\mathrm{RK}^{\bullet}\left(X \times E \mathbb{T}, p_{1}^{*}(H)\right)=\mathrm{RK}_{\bullet}\left(\mathrm{CT}\left(X \times E \mathbb{T}, p_{1}^{*}(H)\right)\right) .
$$

Since $\mathrm{CT}\left(X \times E \mathbb{T}, p_{1}^{*}(H)\right)=\mathrm{CT}(X, H) \otimes C(E \mathbb{T})$ and $C(E \mathbb{T})$ is contractible, by the homotopy invariance of representable K-theory in property (1) above,

$$
\mathrm{RK}^{\bullet}\left(X \times E \mathbb{T}, p_{1}^{*}(H)\right) \cong \mathrm{RK}^{\bullet}(X, H)
$$

and by property (2) above, we see that

$$
\mathrm{RK}^{\bullet}(X, H) \cong \mathrm{K}^{\bullet}(X, H)
$$

Combining the observations above, we see that

$$
\mathrm{K}^{\bullet}(X, H) \cong \mathrm{RK}^{\bullet}\left(X \times E \mathbb{T}, p_{1}^{*}(H)\right)
$$

By the Connes-Thom isomorphism for $\sigma-C^{*}$-algebras [25],

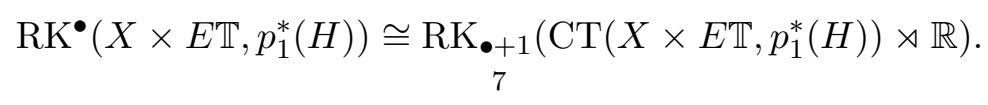


Observe that one has the commutative diagram of finite dimensional approximations of (1),

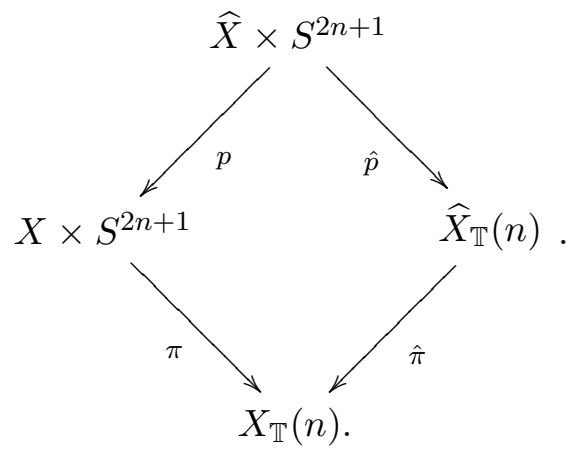

By [26], one has

$$
\mathrm{CT}\left(X \times S^{2 n+1}, p_{1}^{*}(H)\right) \rtimes \mathbb{R} \cong \mathrm{CT}\left(\widehat{X}_{\mathbb{T}}(n), \widehat{H}(n)\right)
$$

and since

$$
\begin{gathered}
\mathrm{CT}\left(\widehat{X}_{\mathbb{T}}, \widehat{H}\right)={\underset{\leftarrow}{n}}_{\lim _{n}} \mathrm{CT}\left(\widehat{X}_{\mathbb{T}}(n), \widehat{H}(n)\right), \\
\mathrm{CT}\left(X \times E \mathbb{T}, p_{1}^{*}(H)\right) \rtimes \mathbb{R}={\underset{\leftarrow}{n}}_{\lim } \mathrm{CT}\left(X \times S^{2 n+1}, p_{1}^{*}(H)\right) \rtimes \mathbb{R},
\end{gathered}
$$

we deduce that

$$
\mathrm{CT}\left(X \times E \mathbb{T}, p_{1}^{*}(H)\right) \rtimes \mathbb{R} \cong \mathrm{CT}\left(\widehat{X}_{\mathbb{T}}, \widehat{H}\right)
$$

and hence that

$$
\mathrm{K}^{\bullet}(X, H) \cong \mathrm{RK}^{\bullet+1}\left(\widehat{X}_{\mathbb{T}}, \widehat{H}\right) .
$$

Next, the $I(\mathbb{T})$-adic completion $\mathrm{K}_{\mathbb{T}}^{\bullet}(\widehat{X}, \widehat{H})^{\sim}$ of twisted equivariant K-theory (where $I(\mathbb{T})$ denotes the augmentation ideal) is isomorphic to, cf. [27],

$$
\mathrm{K}_{\mathbb{T}}^{\bullet}(\widehat{X}, \widehat{H})^{\sim} \cong \mathrm{RK}^{\bullet}\left(\widehat{X}_{\mathbb{T}}, \widehat{H}\right)
$$

Therefore using (3) and (4) we get

$$
\mathrm{K}^{\bullet}(X, H) \cong \mathrm{K}_{\mathbb{T}}^{\bullet}(\widehat{X}, \widehat{H})^{\sim}
$$

which is interpreted as T-duality isomorphism of charges of RR-fields in K-theory.

\section{REFERENCES}

[1] T. Buscher, A symmetry of the string background field equations, Phys. Lett. B194 (1987) 59-62; Path integral derivation of quantum duality in nonlinear sigma models, ibid., B201 (1988) 466-472.

[2] E. Alvarez, L. Alvarez-Gaumé, J.L.F. Barbón and Y. Lozano, Some global aspects of duality in string theory, Nucl. Phys. B415 (1994) 71-100, arXiv:hep-th/9309039.

[3] M.J. Duff, H. Lü and C.N. Pope, $\mathrm{AdS}_{5} \times S^{5}$ untwisted, Nucl. Phys. B532 (1998) 181-209, arXiv:hep-th/9803061.

[4] K. Dasgupta, G. Rajesh and S. Sethi, M theory, orientifolds and G-flux, JHEP 08 (1999) 023, arXiv:hep-th/9908088v3]. 
[5] S. Gurrieri, J. Louis, A. Micu and D. Waldram, Mirror symmetry in generalized Calabi-Yau compactifications, Nucl. Phys. B654 (2003) 61-113, arXiv:hep-th/0211102.

[6] S. Kachru, M. Schulz, P. Tripathy and S. Trivedi, New supersymmetric string compactifications, J. High Energy Phys. 03 (2003) 061, arXiv:hep-th/0211182.

[7] P. Bouwknegt, J. Evslin and V. Mathai, On the Topology and Flux of T-Dual Manifolds, Phys. Rev. Lett. 92 (2004) 181601, hep-th/0312052; T-duality: Topology Change from H-flux, Commun. Math. Phys. 249 (2004) 383-415, arXiv:hep-th/0306062.

[8] R. Minasian and G. Moore, K-theory and Ramond-Ramond charge, J. High Energy Phys. 11 (1997) 002, arXiv:hep-th/9710230.

[9] E. Witten, D-Branes and K-Theory, J. High Energy Phys. 12 (1998) 019, arXiv:hep-th/9810188.

[10] P. Bouwknegt and V. Mathai, D-branes, B-fields and twisted K-theory, J. High Energy Phys. 03 (2000) 007 , arXiv:hep-th/0002023.

[11] U. Bunke and T. Schick, T-duality for non-free circle actions, in: B. Booß-Bavnbek et al (eds.), Analysis, geometry and topology of elliptic operators, pp. 429-466, World Sci. Publ., Hackensack, NJ, 2006, arXiv:math/0508550.

[12] A.S. Pande, Topological T-duality and Kaluza-Klein monopoles, Adv. Theor. Math. Phys. 12 (2008) 185-215, arXiv:math-ph/0612034.

[13] M. Goresky and R. MacPherson, Intersection homology theory, Topology 19 (1980) 135-162.

[14] R.D. Sorkin, Kaluza-Klein Monopole, Phys. Rev. Lett. 51 (1983) 87-90.

[15] K. Hori, S. Katz, A. Klemm, R. Pandharipande, R. Thomas, C. Vafa, R. Vakil and E. Zaslow, Mirror symmetry, Clay Mathematics Monographs, 1. American Mathematical Society, Providence, RI and Clay Mathematics Institute, Cambridge, MA, 2003.

[16] P. Bouwknegt, K. Hannabuss and V. Mathai, T-duality for principal torus bundles, J. High Energy Phys. 03 (2004) 018, arXiv:hep-th/0312284.

[17] M.F. Atiyah and R. Bott, The moment map and equivariant cohomology, Topology 23 (1984) 1-28.

[18] A. Hattori and T. Yoshida, Lifting compact group actions in fiber bundles, Japan J. Math. (N.S.) 2 (1976) 13-25.

[19] I. Mundet i Riera, Lifts of smooth group actions to line bundles, Bull. London Math. Soc. 33 (2001) 351-361, arXiv:math/0002123.

[20] V. Guillemin, V. Ginzburg and Y. Karshon, Moment maps, cobordisms, and Hamiltonian group actions, Math. Surveys and Monographs, 98, Amer. Math. Soc., Providence, RI, 2002.

[21] M. Harada and G. Landweber, The K-theory of abelian symplectic quotients, Math. Res. Lett. 15 (2008) 57-72, arXiv:math.SG/0612660.

[22] K. Hori, D-branes, T-duality, and index theory, Adv. Theor. Math. Phys. 3 (1999) 281-342, arXiv:hep-th/9902102.

[23] N.C. Phillips, Representable K-theory for $\sigma$ - $C^{*}$-algebras, K-Theory 3 (1989) 441-478.

[24] J. Rosenberg, Continuous-trace algebras from the bundle theoretic point of view, J. Austral. Math. Soc. A 47 (1989) 368-381.

[25] N.C. Phillips and L. Schweitzer, Representable K-theory of smooth crossed products by $\mathbb{R}$ and $\mathbb{Z}$, Trans. Amer. Math. Soc. 344 (1994) 173-201.

[26] I. Raeburn and J. Rosenberg, Crossed products of continuous-trace $C^{*}$-algebras by $C^{\infty}$ actions, Trans. Amer. Math. Soc. 305 (1988) 1-45.

[27] N.C. Phillips, The Atiyah-Segal completion theorem for $C^{*}$-algebras, K-Theory, 3 (1989) 479-504. 
(V Mathai) Department of Pure Mathematics, University of Adelaide, Adelaide, SA 5005, Australia E-mail address: mathai.varghese@adelaide.edu.au

(S Wu) Department of Mathematics, University of Hong Kong, Pokfulam Road, Hong Kong, China E-mail address: swu@maths.hku.hk 